\title{
UTILIZACIÓN DE EYE TRACKING PARA EVALUAR EL USO DE INFORMACIÓN VERBAL EN MATERIALES MULTIMEDIA
}

\section{USING EYE TRACKING TECHNIQUES TO EVALUATE THE USE OF VERBAL INFORMATION IN MULTIMEDIA MATERIALS}

\author{
Óscar Navarro Martínez ${ }^{1}$ \\ oscarnavarromartinez@gmail.com \\ Ana Isabel Molina Díaz ${ }^{2}$ \\ AnaIsabel.Molina@uclm.es \\ Miguel Lacruz Alcocer ${ }^{3}$ \\ Miguel.Lacruz@uclm.es \\ ${ }^{I}$ CP San José de Calasanz. Equipo de Orientación \\ Calle Soria, s/n, Tomelloso, Ciudad Real (España) \\ ${ }^{2}$ Universidad de Castilla-La Mancha. E.S. de Informática. Departamento de Tecnologías y \\ Sistemas de Información. Paseo de la Universidad, 4, Ciudad Real (España) \\ ${ }^{3}$ Universidad de Castilla-La Mancha. Facultad De Educación. Departamento de \\ Pedagogía. Ronda de Calatrava, 3, Ciudad Real (España)
}

En el presente trabajo se pretende identificar qué formatos favorecen una mayor eficiencia en el aprendizaje, para así establecer unas pautas de diseño (guidelines) respecto a la distribución y modalidad de presentación de información verbal en materiales educativos multimedia. Se realizaron dos experimentos que utilizaron la técnica de seguimiento ocular (eye tracking) como elemento innovador en el proceso de recogida de datos. Se produce una mayor eficiencia en el aprendizaje de los alumnos cuando los textos y las imágenes relacionadas se presentan próximos en el espacio y cuando la información se proporciona a través de una narración oral.

Palabras clave: material didáctico, recursos multimedia, eye tracking, aprendizaje verbal, Educación Primaria.

In this work that aims to identify which are favor greater efficiency in learning, so as establish a design guidelines respect about distribution and form of verbal presentation in multimedia educational materials. Tow experiments does, which used eye tracking technique how innovation element in the date collection process. Greater efficiency in pupils when the texts and implicate images are presented next in the space and the information proved through storytelling

Keywords: teaching materials, multimedia resources, eye tracking, verbal learning, primary education.

Píxel-Bit. Revista de Medios y Educación. No 48 Enero 2016. ISSN: 1133-8482. e-ISSN: 2171-7966. doi: http://dx.doi.org/10.12795/pixelbit.2016.i48.04 


\section{Introducción.}

En educación se abren nuevos horizontes en el proceso de enseñanza y aprendizaje de los alumnos, con una utilización de recursos y materiales multimedia cada vez más habitual. Los avances tecnológicos han hecho posible la combinación de distintas formas de presentación de información verbal (por ejemplo, la narración y texto en pantalla) con modos de presentación no verbales (como gráficos, vídeos, animaciones y sonidos) en un solo dispositivo (Moreno \& Mayer, 1999).

A la hora de elaborar recursos y materiales multimedia, se debe poner especial énfasis en el proceso de diseño, para que éstos sean efectivos y eficientes para conseguir los objetivos planteados. Nuestro trabajo se llevó a cabo en la etapa de Educación Primaria, y se centró en evaluar distintas configuraciones de presentación de materiales multimedia. Se han diseñado dos experiencias en las que se incluye información verbal en diferentes formatos y en las que se busca conocer cómo analizan la información visual niños de segundo y sexto curso (7 y 11 años respectivamente) de esta etapa educativa. Los resultados de estos experimentos servirán para establecer unas pautas (empíricamente contrastadas) de diseño de materiales educativos multimedia que incluyen contenido en formato texto e imágenes. En dichas experiencias se pretende, a su vez, contrastar algunos de los más conocidos principios para el diseño de materiales multimedia. En particular se tendrán en cuenta las aportaciones de Richard Mayer, que propone una serie de principios de aprendizaje multimedia (Mayer, 2003, 2005, 2010) basados en el modo en que las personas asimilamos este tipo de información. En función del formato de presentación de textos o imágenes se puede favorecer la asimilación de los contenidos o, por el contrario, añadir una carga cognitiva innecesaria. En particular se intentarán testear los principios de Contigüidad Espacial (Spatial Contiguity Principle) y Modalidad (Modality Contiguity Principle) propuestos por Mayer. El primero afirma que las personas aprenden mejor cuando las palabras e imágenes relacionadas se presentan más próximas unas de otras en la pantalla. En la misma línea de este principio, encontramos el Principio de Proximidad de la Gestalt, el cual postula que la distancia relativa entre los objetos de la pantalla afecta a nuestra percepción de los mismos, de forma que aquellos que aparecen próximos, se perciben como relacionados o pertenecientes a una misma categoría o grupo (Ware, 2008). Respecto al principio de Modalidad, indica que cuando se proporciona información tanto gráfica como verbal, se favorece el aprendizaje de los alumnos si la información verbal se proporciona en formato audio y no de manera escrita. Podemos encontrar estudios anteriores que analizan, tanto el Principio de Contigüidad Espacial (Holsanova, Holmberg \& Holmqvist, 2006), como el de Modalidad, con adultos (Mayer, 2010; Moreno \& Mayer, 1999) o con niños de edades similares a las de este estudio (Moreno \& Durán, 2004).

En la realización de los estudios empíricos realizados se recurre a la utilización de una técnica novedosa, como es la técnica de seguimiento ocular (en inglés, eye tracking). Las técnicas de rastreo del movimiento y generación de fijaciones oculares permiten inferir información sobre la atención y el comportamiento de exploración visual de los usuarios. A través de estos dispositivos es posible registrar las llamadas fijaciones (o puntos de estabilización de la mirada) que 
realiza un individuo al observar un contenido mostrado en una pantalla. Posteriormente, se calculan una serie de métricas a partir de las fijaciones generadas para una determinada zona de la pantalla (a la que se conoce como área de interés, $\mathrm{AOI})$. Las AOIs son las partes de la pantalla de las cuales queremos analizar la atención visual de los usuarios (esto es el tiempo que dedican a mirar dicha área o las veces que la consultan). Puesto que queremos evaluar contenido multimedia, se crearán AOIs asociadas a las áreas de la pantalla que contienen la información más relevante o que se pretende transmitir mediante las imágenes y textos mostrados en la presentación multimedia. Podemos encontrar diversos ámbitos o sectores en los que se han empleado técnicas de eye tracking, entre los que destacamos la publicidad y la evaluación de interfaces de usuario y páginas web (Nielsen \& Pernice, 2010; Poole \& Ball, 2005) mientras que en el ámbito educativo el número de estudios es inferior y menos aún en la etapa de Educación Primaria (Mason, Tornatora \& Pluchino, 2013).

Referido a niños de estas edades (7 y 11 años), la investigación abre un importante abanico de posibilidades, pues ya han accedido a la lectoescritura y resulta interesante conocer el escaneo visual que realizan entre la información verbal y la pictórica, estando ésta semánticamente relacionada (Holsanova et al., 2006). En cuanto a los libros de texto que utilizan los niños y niñas en esta etapa educativa, tiene gran relevancia analizar cómo se divide la atención entre textos e ilustraciones (Hannus \& Hyönä, 1999; Hyönä, Lorch \& Rinck, 2003).

La incorporación de la técnica de rastreo ocular, puede ser un complemento ideal a la utilización de otras técnicas de recogida de datos clásicas (Hyönä, 2010; Pretorius, Calitz $\&$ Greunen, 2005) que se han propuesto para analizar la eficiencia de materiales multimedia. El potencial de la incorporación de esta fuente adicional de información está en su naturaleza objetiva. Frente a los cuestionarios o encuestas, que tienen un carácter más subjetivo, la técnica de eye tracking aporta información objetiva, no controlada conscientemente por los sujetos. Resulta de especial interés y utilidad incorporar esta fuente de datos en el caso de los niños. A continuación se comenta la finalidad del estudio empírico descrito en este trabajo, incluyendo los distintos materiales y recursos empleados. Se contextualizará el uso que se realiza de las distintas métricas utilizadas para, finalmente, analizar los resultados de cada uno de los dos experimentos realizados. Se comentarán los resultados de cada experimento por separado, pasando a discutir posteriormente unas conclusiones conjuntas. El objetivo último, tal y como se ha comentado, es proponer unas pautas o recomendaciones (guidelines) a tener en cuenta en el diseño de materiales educativos multimedia destinados a alumnos de Educación Primaria, centrándonos en este caso en la inclusión de información visual y verbal.

\section{Metodología.}

El presente trabajo tiene como finalidad examinar el proceso de exploración visual que realizan los alumnos de las imágenes y textos incluidos en presentaciones multimedia. Se pretende identificar qué formatos favorecen una mayor eficiencia en el aprendizaje, y así establecer unas pautas de diseño (o guidelines) respecto a la distribución o modalidad de presentación de la información 
verbal en la elaboración de materiales educativos. Se diseñaron dos experiencias en las que se mostraba a los alumnos una serie de presentaciones multimedia que incorporaban imágenes y texto. Durante la actividad se hacía uso de un dispositivo de seguimiento ocular (eye tracker) que registraba el comportamiento de exploración visual de los alumnos. En el diseño de la presente investigación se creó un Grupo Experimental (Grupo 1), con alumnos a los que se les mostraría contenido multimedia en un formato que, en teoría, debería facilitar un aprendizaje más eficaz de los contenidos mostrados (en su diseño se tenían en cuenta los principios de diseño propuestos por Richard Mayer, así como las leyes de la Gestalt). Por otro lado, se creó un Grupo de Control (Grupo 2), al que se les mostraban materiales con un formato que no seguía estas directrices.

Nuestro objetivo final consistía en establecer una serie de pautas de diseño (guidelines) respecto a la distribución y modalidad de presentación de información verbal en la elaboración de materiales multimedia. Las hipótesis que se pretenden testear en estos estudios son las siguientes:

$\mathbf{H}_{\mathbf{1}}$ : Se producirá una mayor eficiencia en la retención de contenidos si textos e imágenes se muestran próximos en el espacio (en pantalla), en vez de separados.

$\mathbf{H}_{2}$ : Se producirá una mayor eficiencia en la asimilación de contenidos si la explicación asociada a dichas imágenes se realiza mediante narración oral, en vez de textos escritos.

\subsection{Participantes.}

En el estudio empírico han intervenido un total de 49 alumnos pertenecientes a la etapa de Educación Primaria de un centro de Tomelloso (Ciudad Real), 25 de ellos de segundo curso (cuya edad media era $\mathrm{M}=7.55$, $\mathrm{SD}=0.29)$ y 24 de sexto curso $(\mathrm{M}=11.72$, $\mathrm{SD}=0.41)$. Todos los participantes conocen perfectamente el castellano pues, aunque algunos son hijos de inmigrantes, han nacido en España y han estado escolarizados desde los tres años. Se seleccionaron los alumnos de segundo y sexto curso de Educación Primaria por mostrar unas diferencias psicoevolutivas significativas en el tratamiento de textos. Antes de realizar los experimentos se realizó una prueba piloto con 8 alumnos para determinar la duración de las presentaciones y hacer pruebas de calibración del eye tracker. Principalmente se hicieron pruebas con alumnos de segundo curso, ya que presentan más problemas a la hora de comprender o atender órdenes concretas. En algún caso se eliminaron los datos de algunos alumnos, ya que hubo problemas en la recogida de información.

\subsection{Materiales.}

Las presentaciones mostradas a los alumnos están compuestas de dos o tres diapositivas con imágenes, textos, así como grabaciones de audio adaptadas al nivel de comprensión de los alumnos. Dichos contenidos son muy similares a los utilizados con alumnos de esta edad en el centro educativo y en el ámbito de la enseñanza de las Matemáticas.

Podemos encontrar otros estudios similares que también centran su investigación en aprendizajes en esta área de conocimiento (Andrà et al., 2009; García, 2008; Moreno \& Durán, 2004), aunque ninguno de ellos utiliza técnicas de seguimiento ocular en sus análisis. Los contenidos 


\section{Experimento $1 \quad$ Experimento 2}
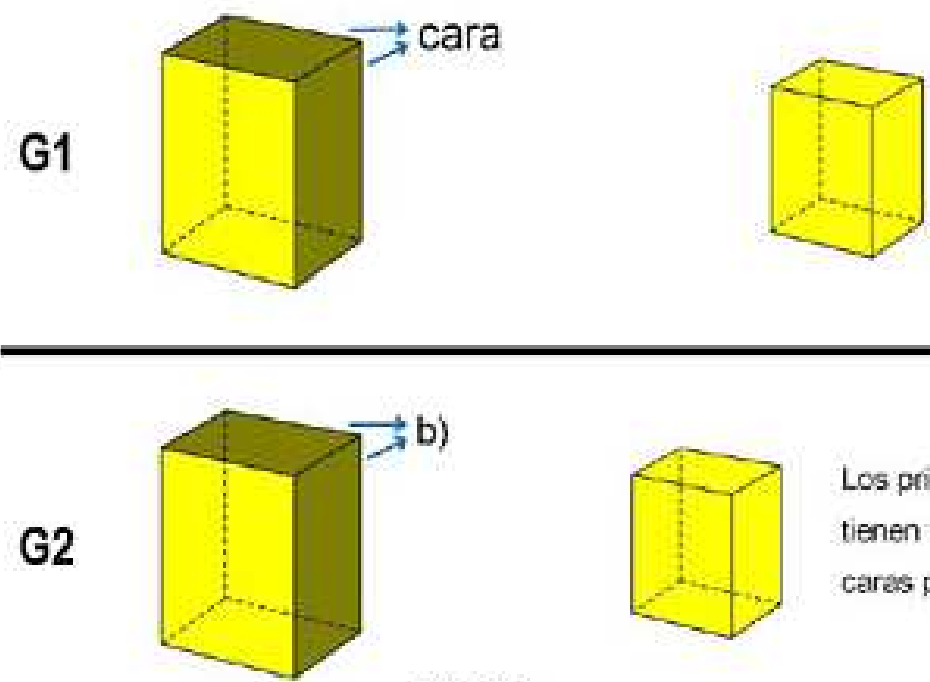

Los prismas

tienen todas sus

cares planas

\section{b) cara}

Figura 1. Extracto de las presentaciones mostradas a los alumnos de segundo curso

seleccionados corresponden al tercer trimestre, por lo que todavía no han sido impartidos, y son desconocidos por los estudiantes. En la Figura 1 podemos ver un ejemplo de algunos extractos de las presentaciones que se mostraron a los alumnos de segundo curso. La mostrada en la parte superior pertenece al Grupo 1, que se corresponde con el Grupo Experimental. En la parte inferior de la figura observamos la pantalla mostrada al Grupo de Control, que ofrece la misma información, pero en distinto formato.

\subsection{Dispositivo.}

Para el registro de los movimientos oculares de los niños y niñas se utilizó un dispositivo
Tobii X60, fabricado por Tobii Technology (Tobii, 2014). Dicho dispositivo funciona a una frecuencia de muestreo de $60 \mathrm{~Hz}$ y tiene una resolución espacial de menos de $0,5^{\circ}$. El eye tracker se acopló a un monitor TFT de 24 pulgadas con una resolución máxima de 1920 x 1200 pixeles. El sistema permite amplios movimientos de la cabeza, proporcionando un entorno de prueba libre de distracciones que asegura un comportamiento natural, y por lo tanto, resultados válidos. La alta exactitud y precisión de la tecnología de seguimiento asegura que los resultados de la investigación sean fiables (Tobii pro, 2014). El monitor se debe situar aproximadamente a sesenta centímetros de los ojos. Dada la diferencia de altura de los alumnos se utilizaron tres sillas adaptables, lo cual permitía 
situar a cada alumno a la distancia y altura correcta. Podemos encontrar diversas investigaciones que han utilizado un dispositivo y software similar con mayor o menor presencia de elementos multimedia (Aranyanak \& Reilly, 2013; Koski, Olson \& Newcombe, 2013; Lagun, Hsieh, Webster \& Navalpakkam, 2014; Mason et al., 2013; Molina, Redondo, Lacave \& Ortega, 2014; Walber, Neuhaus, Staab, Scherp \& Jain, 2013).

\subsection{Procedimiento.}

En la Figura 2 se muestra el diseño experimental de este trabajo. El estudio se realizó en distintas dependencias del centro educativo. En primer lugar se aplicó individualmente el Test Breve de Inteligencia de Kaufman (K-BIT) (Kaufman \& Kaufman, 2011), cuyo objetivo es medir la inteligencia verbal y no verbal de los alumnos. En la realización de esta prueba, los alumnos se ausentaban de su aula durante unos 15 minutos aproximadamente. Posteriormente se realizó el Inventario de Estilos de Aprendizaje de Felder para conocer el estilo de aprendizaje de cada alumno (Felder \& Silverman, 1988; Litzinger, Lee, Wise \& Felder, 2007). Éste se cumplimentó en el aula correspondiente de cada curso, durante una sesión completa en el caso de los alumnos de sexto curso (45 minutos), y una sesión y media aproximadamente en el caso de los de segundo curso (70 minutos). Con estos datos se realizó el muestreo que permitía la creación de dos grupos homogéneos. Posteriormente

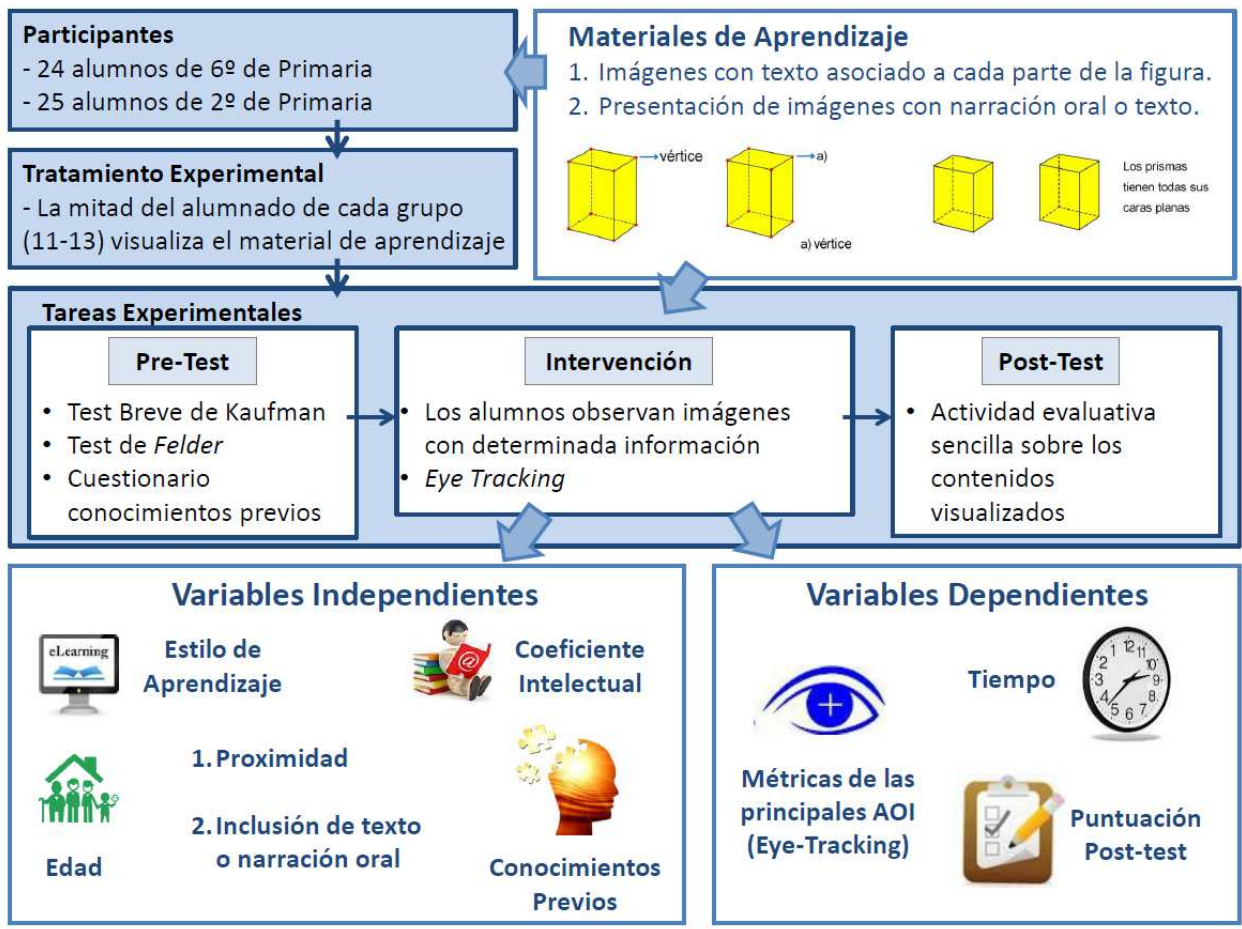

Figura 2. Diseño experimental 
se usó el aula de informática para la realización de las tareas experimentales y la recogida de datos. De forma individual los alumnos realizaban primero un pretest, compuesto de una serie de preguntas abiertas, con el que se pretendía determinar sus conocimientos previos sobre los contenidos que posteriormente se les iban a mostrar. Seguidamente se situaba el alumno frente al monitor y se realizaba la calibración del eye tracker. A continuación se mostraban en pantalla las distintas diapositivas de la presentación, pasando de una a otra utilizando el ratón cuando el alumno lo consideraba conveniente. Mientras realizaban el estudio de los materiales mostrados, el eye tracker registraba las fijaciones realizadas por los niños durante la actividad. Por último, y una vez finalizada la prueba, los alumnos debían cumplimentar otro cuestionario, que pretendía medir el nivel de contenidos asimilados. El tiempo total requerido para completar todas las fases por parte de cada alumno fue de unos 30 minutos aproximadamente.

Las actividades evaluativas que se propusieron a los niños y niñas tenían la misma estructura y características que las realizadas habitualmente por ellos en el centro educativo, por tanto, los alumnos que participaron estaban familiarizados con tareas de este tipo, por lo que no existía ninguna dificultad añadida que pudiera interferir en los resultados obtenidos. Por su parte, previamente a la presentación de los materiales se dieron las indicaciones necesarias, siempre en el mismo orden y con un lenguaje sencillo, especialmente en el caso de los alumnos de segundo curso, dado que se encuentran en un estadio evolutivo diferente a los de sexto y requieren un trato distinto. Igualmente, para los alumnos de menor edad se incluyó un menor número de palabras en los textos, con una fuente de texto e imágenes de mayor tamaño.

\subsection{Análisis de datos.}

Los resultados obtenidos en el pretest, así como las métricas proporcionadas por el eye tracker fueron exportados a Excel y SPSS para realizar el análisis estadístico correspondiente. Se realizó un contraste unilateral de medias en la verificación de las hipótesis enunciadas. En primer lugar se efectuó el análisis de varianzas y posteriormente el cálculo de la t de Student. En apartados posteriores se muestran gráficos comparativos con los datos obtenidos en el posttest realizado después de ver las presentaciones, así como el tiempo total empleado por los alumnos en dichas observaciones. También se ilustra cómo se ha producido la atención visual de los alumnos de ambos grupos, comparando las densidades de fijación, mediante los llamados mapas de calor o heatmaps obtenidos. Basándonos en la bibliografía existente sobre el tema (Birkett, Galpin, Cassidy, Marrow, \& Norgate, 2011; Bojko, 2013; Jacob \& Karn, 2003; Mason et al., 2013; Poole \& Ball, 2005) podemos encontrar gran variedad de métricas que se han utilizado habitualmente en sesiones de eye tracking para medir aspectos como la atención, la complejidad, o la utilidad de la información contenida en una determinada área de interés (AOI). En función del dispositivo y el software utilizado podemos calcular distintas métricas. Sin embargo, el principal inconveniente es la diversidad y cantidad de ellas que se pueden utilizar, no habiendo unanimidad sobre la conveniencia de emplear unas u otras (Hyönä et al., 2003). 


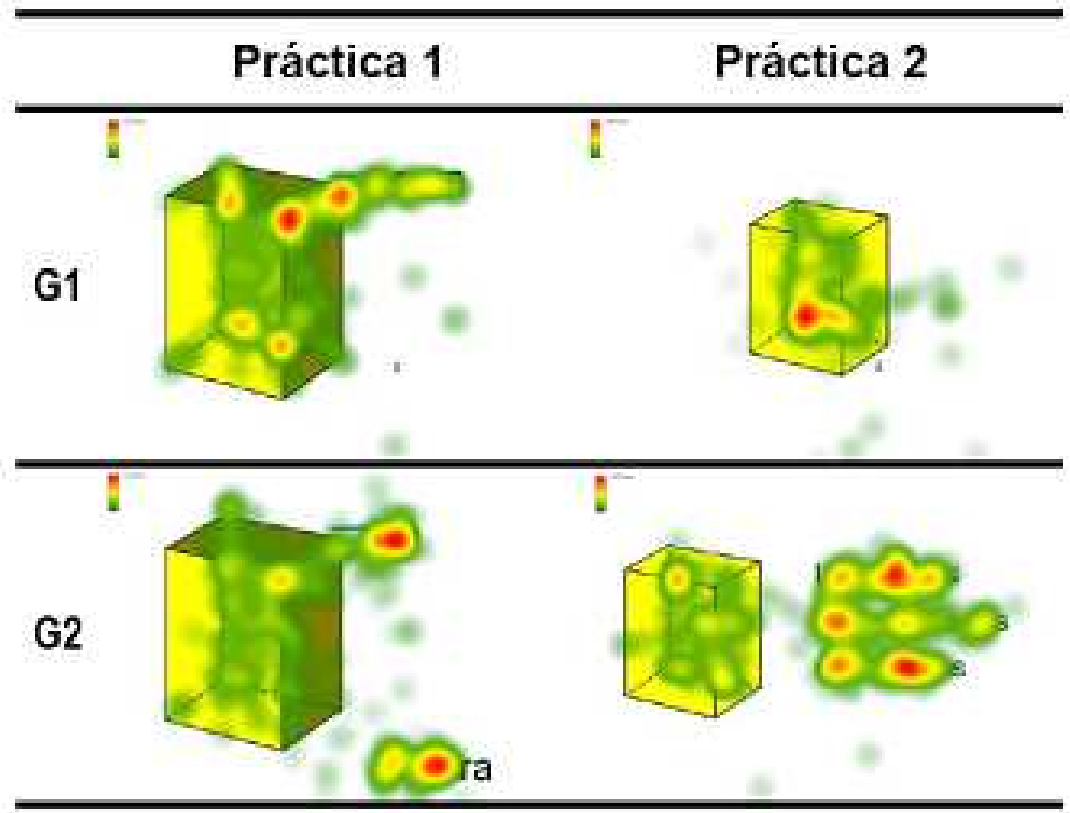

Figura 3. Parte de las presentaciones mostradas a los alumnos de segundo curso

Para este trabajo, se ha recurrido a una serie de métricas que registran cómo y cuándo se focaliza la mirada en determinadas áreas (AOIs) que tienen especial relevancia para nuestra investigación (en este caso el texto o las imágenes mostradas en pantalla). En primer lugar mencionamos el tiempo de duración hasta la primera fijación en un área de interés (Time to First Fixation - TFF) y el número de fijaciones antes de centrar la mirada en un AOI (Fixations Before - FB), que indicarán la antelación o anticipación con la que se fija la atención en una zona concreta de la pantalla. Estas dos métricas se especifican para las imágenes (Im), el texto (Tx) y la medición conjunta de ambas (Total). También se analizaron otras como el número total de fijaciones en la pantalla (FC-Sc) y la proporción de fijaciones en una determinada AOI (FC/FC-Sn). El dispositivo de seguimiento ocular también podía registrar otras métricas, como la duración hasta que se enfoca la mirada la primera vez, el número y duración de todas las fijaciones en una AOI el número y duración de las visitas realizadas en cada AOI, pero no se tuvieron en cuenta por no aportar información relevante en la presente investigación, ya que sólo se seleccionaron las que tienen una relación directa con la eficiencia en el aprendizaje.

En los dos experimentos realizados se establecieron dos grupos para cada uno de los niveles educativos analizados, segundo y sexto de la etapa de Educación Primaria. El Grupo 1 corresponde al Grupo Experimental, al que se aplican las configuraciones que favorecen el aprendizaje por parte de los alumnos. Por otro lado, el Grupo 2 se identifica con el Grupo de Control. 


\section{Posttest}

Práctica 1

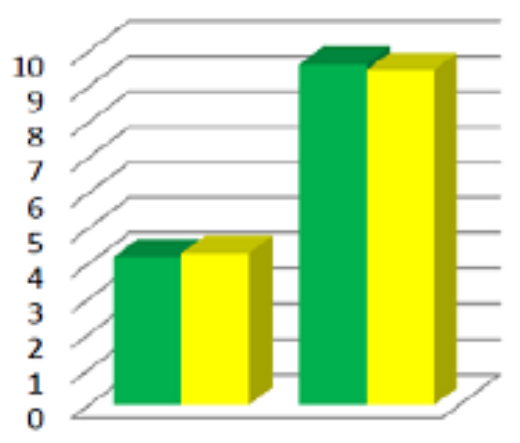

22

Grupo 1 Grupo 2

\section{Tiempo total \\ Práctica 1}

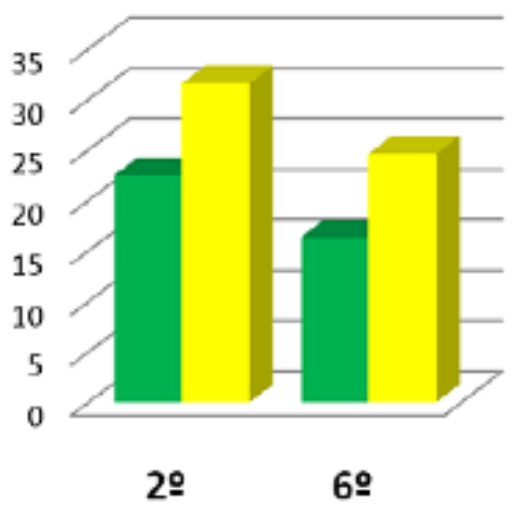

Grupo 1 Grupo 2

Figura 4. Resultados del posttest y tiempo total. Experimento 1

\section{Resultados.}

A continuación se exponen los resultados de las dos experiencias realizadas. Se incluirán valores referentes a los posttest realizados por los alumnos y los datos registrados por el eye tracker. Es necesario definir las áreas de interés (AOI), para valorar posteriormente la observación que realizan ambos grupos sobre las mismas. Dichas AOIs harán referencia a las imágenes y al texto (en los casos en que aparezca).

En un primer momento se pueden analizar algunas representaciones, con un carácter más cualitativo, como son los mapas de calor (o heatmaps), mostrados en la Figura 3. Las «zonas calientes» (marcadas en rojo) señalan la zona de la pantalla en la cual han centrado más la atención los alumnos. En este extracto se observa claramente, en ambos experimentos, cómo se realiza una mayor observación por parte de los usuarios del Grupo 1 (experimental) en las zonas de la imagen más relevantes (caras del prisma). Se valora además la eficiencia de las presentaciones en base a los datos obtenidos en el posttest y la medida de la duración total del tiempo que permanecen los niños observando la pantalla, para a continuación contrastar estas conclusiones con las métricas registradas por el eye tracker (con especial atención en la diferenciación de textos e imágenes).

\subsection{Proximidad de texto e imágenes.}

El Grupo 1, Experimental (verde), representa a los alumnos a los que se les muestran imágenes con texto muy próximo en el espacio, frente a los del Grupo 2, de Control

Píxel-Bit. Revista de Medios y Educación. No 48 Enero 2016. ISSN: 1133-8482. e-ISSN: 2171-7966. doi: http://dx.doi.org/10.12795/pixelbit.2016.i48.04 
(amarillo), para los que se han situado las imágenes en la misma posición que en la otra configuración y los textos en la zona inferior de la pantalla. La puntuación obtenida para segundo curso en el posttest es prácticamente idéntica en ambos grupos (Figura 4). Esta igualdad se confirma al realizar el contraste unilateral de medias, en el que se obtienen valores de la $t$ de Student inferiores al valor crítico, por lo que no se encuentran diferencias significativas. Sin embargo observamos una clara diferencia en el tiempo empleado por los niños y niñas para mirar la pantalla, con una menor duración para el Grupo Experimental. Para el tiempo de observación, con un nivel de significación de 0,05 , obtenemos un valor de $t=2,25$ $(\mathrm{p}=0,015)$, mayor que el valor crítico. En sexto curso obtenemos datos similares, como en el caso anterior los resultados del posttest son casi iguales y no aparecen diferencias significativas. También podemos afirmar que el tiempo utilizado por el Grupo Experimental es significativamente menor, con $\mathrm{t}=3,02$ ( $\mathrm{p}=0,002)$ y un nivel de significación de 0,01 .

Respecto a las métricas proporcionadas por el eye tracker, en segundo curso se observan diferencias en varias de ellas, con distintos niveles de significación. Los alumnos del Grupo 1 tardan menos tiempo en fijar la mirada en cualquiera de las AOI (TFF-All), con valores de $\mathrm{t}=1,88(\mathrm{p}=0,034)$, y asimismo, realizan menos fijaciones antes de enfocar la mirada (FB), tanto en imágenes, en textos como en total, con valores de $\mathrm{t}=1,49(\mathrm{p}=0,072)$, $\mathrm{t}=1,43 \quad(\mathrm{p}=0,081)$ y $\mathrm{t}=3,05 \quad(\mathrm{p}=0,002)$ respectivamente. Además, los alumnos del Grupo 1 realizan un menor número de fijaciones totales en pantalla (FC-Sc), con $\mathrm{t}=2,09(\mathrm{p}=0,022), \mathrm{y}$ una mayor proporción de fijaciones en las AOIs (FC/FC-Sc), con t=5,987 $(\mathrm{p}<0,001)$. En cuanto a las métricas en sexto curso, los alumnos del Grupo 1 tardan menos tiempo en fijar la mirada (TTF) y en un menor número de veces $(\mathrm{FB})$ en los textos, con $\mathrm{t}=2,69(\mathrm{p}=0,005)$ y $\mathrm{t}=3,49 \quad(\mathrm{p}<0,001)$ respectivamente. Además, el número de veces que se fija la mirada teniendo en cuenta ambas AOIs es menor, con $t=2,34(p=0,012)$. Se obtienen resultados muy similares en las dos últimas métricas comentadas para segundo curso. También, los alumnos del Grupo 1 realizan un menor número de fijaciones totales en pantalla ( $\mathrm{FC}-\mathrm{Sc})$, con $\mathrm{t}=2,79(\mathrm{p}=0,004)$, $\mathrm{y}$ una mayor proporción de fijaciones en las AOIs (FC/FC-Sc), con t=7,491 (p<0,001).

La primera conclusión a la que podemos llegar al analizar los resultados es que se obtienen puntuaciones prácticamente idénticas en el posttest en ambos niveles educativos (segundo y sexto), pero se dedica menos tiempo a observar los contenidos en el caso del Grupo Experimental (aquel al que se le mostraba la configuración que teóricamente favorece la asimilación de los aprendizajes). Por lo tanto, se logra en ambos niveles educativos una mayor eficiencia en el aprendizaje cuando se muestran las imágenes y los textos explicativos próximos en el espacio (tal y como enuncian el principio de Contigüidad Espacial de Mayer y el principio de Proximidad de la Gestalt).

Estos resultados se confirman al revisar las métricas registradas por el eye tracker. Los alumnos de segundo curso del Grupo 1 (Experimental) enfocan la mirada en un tiempo inferior y generando un menor número de fijaciones antes de centrar su atención en un AOI, lo cual indica que esta configuración facilita la percepción de los contenidos mostrados por parte de los niños y niñas, ya que se realiza una búsqueda, o exploración visual de los contenidos, más eficiente y directa. A la misma conclusión se llega al 
observar que, para el Grupo 1, se registra un número menor de fijaciones totales en la pantalla y una mayor proporción de fijaciones en las AOI, que indica mayor eficiencia en la percepción visual para este grupo. En el caso de los alumnos de sexto se observa una tendencia similar. Los alumnos del Grupo 1 también enfocan la mirada antes y con un número menor de fijaciones en las AOIs, pero destacando principalmente los textos. De igual forma que en segundo curso, los alumnos del Grupo 1 realizan un menor número de fijaciones totales y una mayor proporción en las AOIs, por lo que se produce también una mayor eficiencia, destacando la observación de los textos para este nivel.

Por tanto, podemos afirmar que en ambos niveles educativos se produce un aprendizaje más eficiente para los alumnos del Grupo Experimental (Grupo 1), ya que se obtiene una puntuación muy similar pero empleando los alumnos del Grupo 2 en ambos niveles más tiempo en observar las presentaciones, un $40 \%$ y un $50 \%$ respectivamente. Además se obtienen en casi todos los casos niveles de significación bastante altos. Destacamos que los alumnos del Grupo 1 de sexto curso consiguen una mayor eficiencia en la localización de los textos que los del Grupo 2.

\subsection{Uso de narración oral o texto escrito.}

El Grupo 1, Experimental (verde), representa a los alumnos a los que se les muestran imágenes acompañadas de narración oral, frente a los del Grupo 2, de Control (amarillo), para los que aparecen estas imágenes acompañadas de texto escrito. La puntuación obtenida para segundo curso en el posttest es superior para los alumnos del Grupo Experimental (Figura 5). Esta diferencia se confirma al realizar el contraste unilateral de medias, en el que se obtienen valores de la $t$ de Student superiores al valor crítico, con $\mathrm{t}=2,207$ ( $\mathrm{p}=0,016$ ), $\mathrm{y}$ un nivel de significación de 0,05 . Además, observamos que hay diferencia en el tiempo empleado por los niños y niñas para observar la pantalla, utilizando menos tiempo el Grupo Experimental, con valor de $\mathrm{t}=2,27(\mathrm{p}=0,014)$, con un nivel de significación de 0,05, obtenemos un valor mayor que el valor crítico. En el caso de sexto curso obtenemos datos similares. Como en el caso anterior los resultados del posttest son mayores para el Grupo Experimental, con un valor de $\mathrm{t}=1,657$ ( $\mathrm{p}=0,053$ ), aunque sólo con un nivel de significación de 0,1 . También podemos afirmar que el tiempo utilizado por el Grupo Experimental es significativamente menor, con un valor de $t=2,421(p=0,01)$ y un nivel de significación de 0,05 .

Respecto a las métricas proporcionadas por el eye tracker, en ambos cursos se observan diferencias en casi todas las medidas registradas con distintos niveles de significación, en la mayoría de los casos de 0,01 . Se tendrán en cuenta solamente algunas de las métricas, pues el hecho de que aparezcan únicamente textos para uno de los grupos (Grupo 2), altera los resultados y no permite realizar una comparación objetiva. No se tienen en cuenta las métricas que se refieren a los textos y a todas las AOIs, salvo algún caso. Los alumnos del Grupo 1 de segundo curso tardan menos tiempo en fijar la mirada en la imágenes (TFF-Im), con un valor de $\mathrm{t}=1,89(\mathrm{p}=0,033)$, y asimismo, realizan menos fijaciones antes de enfocar la mirada en dichas imágenes (FB-Im), con un valor de $\mathrm{t}=2,21(\mathrm{p}=0,016)$. También, los alumnos del Grupo 1 realizan un menor número de fijaciones totales en pantalla (FC-Sc), con $t=4,552(p<0,001)$, y una mayor proporción 


\section{Posttest}

Práctica 2

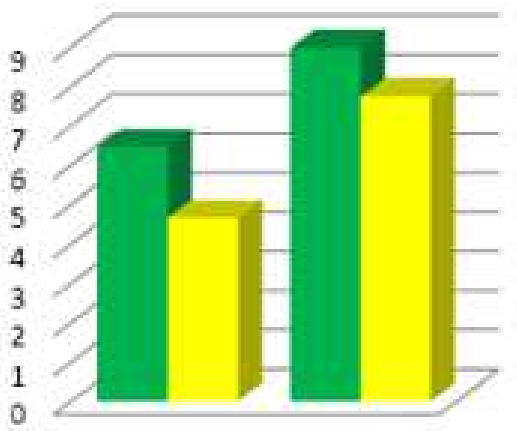

20

\section{Grupo 1 Grupo 2}

\section{Tiempo total}

Práctica 2

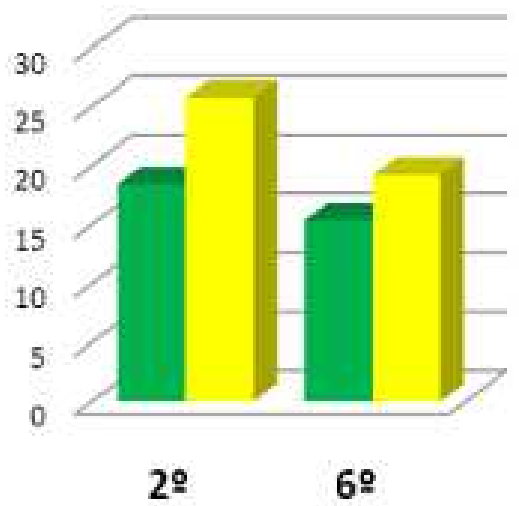

Grupo 1 Grupo 2

Figura 5. Resultados del posttest y tiempo total. Experimento 2

de fijaciones en las imágenes (FC-Im/FC-Sc), con $\mathrm{t}=9,892(\mathrm{p}<0,001)$. En cuanto a las métricas en sexto curso, los resultados son muy similares a los obtenidos en segundo. Los alumnos del Grupo 1 de segundo curso tardan menos tiempo en fijar la mirada en la imágenes (TFF-Im), con un valor de $\mathrm{t}=3,069$ $(\mathrm{p}=0,002)$, y asimismo, realizan menos fijaciones antes de enfocar la mirada (FB) en las imágenes e incluso teniendo en cuenta ambas AOIs, con valores de $\mathrm{t}=3,337(\mathrm{p}=0,001)$ y $\mathrm{t}=2,288(\mathrm{p}=0,014)$ respectivamente. También, los alumnos del Grupo 1 realizan un menor número de fijaciones totales en pantalla (FC-Sc), con $\mathrm{t}=3,873$ ( $\mathrm{p}<0,001)$, y una mayor proporción de fijaciones en las imágenes (FCIm/FC-Sc), con $\mathrm{t}=19,912$ ( $\mathrm{p}<0,001)$.

La primera conclusión a la que se llega al analizar los resultados es que se obtienen puntuaciones mayores en el posttest tanto en segundo como en sexto, y además se dedica menos tiempo para asimilar los contenidos por parte del Grupo Experimental. Por tanto, se logra en ambos niveles educativos una mayor eficiencia en el aprendizaje si aparecen imágenes acompañadas de narración oral, en vez de imágenes y texto escrito (como enuncia el principio de Modalidad de Mayer).

Estos resultados se confirman al revisar las métricas registradas por el eye tracker. Los alumnos de segundo curso del Grupo 1 (Experimental) enfocan la mirada en un tiempo inferior y generando un menor número de fijaciones, antes de centrar su atención en las imágenes, lo cual indica que esta configuración facilita la percepción de los contenidos mostrados por parte de los niños y niñas, ya que se realiza una búsqueda más eficiente y directa para encontrar estas AOIs. 
A la misma conclusión se llega al observar que, para el Grupo 1, se registra un número menor de fijaciones totales en la pantalla y una mayor proporción de fijaciones en las imágenes, lo cual indica mayor eficiencia en la percepción visual de las principales AOI. En el caso de los alumnos de sexto se observa una tendencia similar, aunque varían ligeramente los niveles de significación. Los alumnos del Grupo 1 también enfocan la mirada antes y con un número menor de fijaciones en las imágenes, incluso en el segundo caso teniendo en cuenta todas las AOIs. De igual forma que en segundo curso, los alumnos del Grupo 1 realizan un menor número de fijaciones totales y una mayor proporción en las AOIs, por lo que se produce también una mayor eficiencia.

Por tanto, podemos afirmar que en ambos niveles educativos se produce un aprendizaje más eficiente para los alumnos del Grupo Experimental (Grupo 1), ya que se obtiene una puntuación mayor, y además, los alumnos del Grupo 2 en ambos cursos emplean más tiempo en observar las presentaciones, un $40 \%$ y un $25 \%$ respectivamente. Además se obtienen en casi todos los casos niveles de significación bastante altos.

\section{Discusión.}

El objetivo de este estudio era analizar la eficiencia en el aprendizaje de alumnos de Educación Primaria en tareas de retención de los contenidos mostrados en presentaciones multimedia que combinaban información verbal e imágenes.

Hemos podido comprobar, de forma empírica, como el proceso de exploración del texto varía en función de la edad. A edades tempranas se realiza una observación menos duradera del texto (Evans \& Saint-Aubin,
2005), pues las imágenes captan mejor la atención de los niños, a las que dedican más tiempo (Slykhuis, Wiebe \& Annetta, 2005). Por su parte, un formato que integre texto e ilustraciones, y los muestre de forma contigua en el espacio mejora significativamente la asimilación de la información mostrada (Holsanova et al., 2006). La utilización de una configuración en la que las áreas de interés (texto e imágenes) se muestren más cercanas en el espacio permite que se consiga resultados similares en cuanto al aprendizaje, pero que se dedique menos tiempo a la exploración visual de los contenidos. Y este fenómeno se ha podido observar en dos niveles educativos (segundo y sexto curso de Educación Primaria). Además, este formato facilita una anticipación de la observación y exploración de los contenidos por parte de los niños y niñas, ya que fijan antes la atención en dichas áreas, generando un menor número de fijaciones y, en el caso de los alumnos de sexto curso, en menos tiempo. Este procesamiento visual integrado entre texto y gráficos se asocia con un mejor aprendizaje (Mason et al., 2013) y una mejor asimilación de la relación que existe entre los contenidos mostrados mediante ambos elementos.

Por tanto, y teniendo en cuenta los resultados obtenidos en la primera de las experiencias descritas, se acepta como válida la hipótesis $\mathbf{H}_{1}$, que enuncia que se da una mayor eficiencia en la retención de contenidos si textos e imágenes se muestran próximos en el espacio, en vez de separados.

La segunda experiencia realizada trataba de analizar el uso de dos canales (modalidades) de presentación de la información verbal (audio vs. texto escrito). Hay autores que defienden que una animación o imagen descrita de forma oral, en lugar de texto 
escrito, conduce a mejores resultados de aprendizaje (Van \& Scheiter, 2010). En este trabajo se ha realizado un experimento que pretende testear, de forma empírica, dicha opinión. Los resultados obtenidos en la segunda experiencia realizada nos permiten aceptar la hipótesis $\mathbf{H}_{2}$. En este caso se ha podido observar que se da una mayor eficiencia en la retención de contenidos si la presentación de imágenes se realiza mediante narración oral (canal auditivo), en vez de textos escritos (canal visual), tanto para segundo como sexto curso.

A partir de todos los resultados obtenidos, mediante el posttest y las métricas proporcionadas por el dispositivo eye tracker, podemos enunciar unas primeras pautas de diseño (o guidelines) a aplicar en el diseño de contenidos multimedia destinados a alumnos de Educación Primaria:

- En el diseño de contenidos multimedia que incluyan imágenes y texto explicativo, se recomienda una distribución de los mismos próxima en el espacio, frente a una configuración en la que ambos elementos aparezcan visualmente menos integrados. Tal y como se ha verificado empíricamente, se produce una mayor eficiencia en la retención de contenidos, por parte de los alumnos de Educación Primaria, cuando los contenidos se muestran en este formato.

- En el diseño de contenidos multimedia que incluyan imágenes e información verbal, se recomienda incluir la explicación de las imágenes mediante narración oral (modalidad auditiva) frente a la visualización de texto escrito en pantalla (modalidad visual).

En cuanto a las líneas de trabajo futuro, nos planteamos continuar realizando experimentos similares a los descritos, aunque ampliando la muestra de alumnos. Además nos planteamos continuar testeando empíricamente otros principios de Mayer también relacionados con el uso de textos en presentaciones multimedia. El objetivo último es proporcionar un conjunto de pautas más amplio, dada la importancia de la inclusión de imágenes y/o textos en los materiales educativos destinados a alumnos en la etapa de Educación Primaria.

\section{Apoyos y agradecimientos.}

Los autores quieren agradecer al Grupo CHICO de la UCLM por facilitar el uso del dispositivo Tobii X60, así como al equipo directivo, tutores y alumnos de segundo y sexto curso de Educación Primaria del CEIP San José de Calasanz por haber participado en los experimentos.

\section{Referencias bibliográficas.}

Andrà, C., Arzarello, F., Ferrara, F., Holmqvist, K., Lindström, P., Robutti, O. \& Sabena, C. (2009). How students read mathematical representations: an eye tracking study. In Tzekaki, M., Kaldrimidou, M. \& Sakonidis, C. (Eds.), Proceedings of the 33rd Conference of the International Group for the Psychology of Mathematics Education (vol. 1) (pp. 49-56). Thessaloniki, Greece: PME.

Aranyanak, I. \& Reilly, R. G. (2013). Asystem for tracking braille readers using a Wii Remote and a refreshable braille display. Behavior Research Methods, 45 (1), 216-228. doi: 10.3758/s13428-012-0235-8.

Birkett, S., Galpin, A., Cassidy, S., Marrow, L. \& Norgate, S. (2011). How revealing are eye-movements for understanding web engagement in young children. Proceedings of the International Conference on Human Factors in Computing Systems, May 7-12, 
Vancouver, BC, Canada, 2251- 2256. doi: 10.1145/1979742.1979900

Bojko, A. (2013). Eye tracking the user experience: a practical guide to research. Brooklyn, New York: Rosenfeld Media.

Evans, M. A. \& Saint, J. (2005). What Children Are Looking at During Shared Storybook Reading: Evidence From Eye Movement Monitoring. Psychological Science, 16(11), 913-920. doi: 10.1111/j.14679280.2005.01636.x

Felder, R. M. \& Silverman, L. K. (1988). Learning and Teaching Styles in Engineering Education. Engineering Education, 78 (7), 674-681.

García, C. (2008). Eye Tracking technology applied to the design of safety and health signs at work. En VI Congreso Internacional de Prevención de Riesgos Laborales, 14-16 de Mayo, A Coruña (España).

Hannus, M. \& Hyönä, J. (1999). Utilization of Illustrations during Learning of Science Textbook Passages among Low- and HighAbility Children. Contemporary Educational Psychology, 24 (2), 95-123. doi: 10.1006/ ceps.1998.0987

Holsanova, J., Holmberg, N. \& Holmqvist, K. (2006). Tracing Integration of Text and Pictures in Newspaper Reading. CiteSeerX. Recuperado de http://citeseerx.ist.psu.edu/ viewdoc/download?doi=10.1.1.207.2056\&re $\mathrm{p}=$ rep $1 \&$ type $=$ pdf

Hyönä, J. (2010). The use of eye movements in the study of multimedia learning. Learning and Instruction, 20 (2), 172-176. doi:10.1016/ j.learninstruc.2009.02.013

Hyönä, J., Lorch, R. F. \& Rinck, M. (2003). Eye Movement Measures to Study Global Text Processing. In Hyönä, R. Radach \& H. Deubel (Eds.), The Mind's Eye. Cognitive and Applied Aspects of Eye Movement Research (pp. 313-334). Amsterdam: Elsevier Science.
Jacob, R. J. K. \& Karn, K. S. (2003). Eye tracking in human-computer interaction and usability research: Readi to deliver the promises. In J. Hyona, R. Radach \& H. Deubel (Eds.), The Mind's Eye. Cognitive and Applied Aspects of Eye Movement Research (pp. 573-605). Amsterdam: Elsevier Science.

Kaufman, A. S. \& Kaufman, N. L. (2011). $K$ BIT: test breve de inteligencia de Kaufman. Madrid: Pearson.

Koski, J., Olson, I. R. \& Newcombe, N. S. (2013). Tracking the eyes to see what children remember. Memory, 21(3), 396-407. doi: 10.1080/09658211.2012.735241

Lagun, D., Hsieh, C.-H., Webster, D. \& Navalpakkam, V. (2014). Towards better measurement of attention and satisfaction in mobile search. In Proceedings of the 37th international ACM SIGIR conference on Research \& development in information retrieval (pp. 113-122). ACM Press. doi:10.1145/2600428.2609631

Litzinger, T. A., Lee, S. H., Wise, J. C. \& Felder, R. M. (2007). A Psychometric Study of the Index of Learning Styles. Journal of Engineering Education, 96(4), 309-319. doi: 10.1002/j.2168-9830.2007.tb00941.x

Mason, L., Tornatora, M. C. \& Pluchino, P. (2013). Do fourth graders integrate text and picture in processing and learning from an illustrated science text? Evidence from eyemovement patterns. Computers \& Education, 60 (1), 95-109. doi: 10.1016/ j.compedu.2012.07.011

Mayer, R. E. (2003). The promise of multimedia learning: using the same instructional design methods across different media. Learning and Instruction, 13(2), 125139. doi:10.1016/S0959-4752(02)00016-6

Mayer, R. E. (Ed.). (2005). The Cambridge handbook of multimedia learning. 
Cambridge, U.K., New York: Cambridge University Press.

Mayer, R. E. (2010). Unique contributions of eye-tracking research to the study of learning with graphics. Learning and Instruction, 20 (2),167-171. doi:10.1016/ j.learninstruc.2009.02.012

Molina, A. I., Redondo, M. A., Lacave, C. \& Ortega, M. (2014). Assessing the effectiveness of new devices for accessing learning materials: An empirical analysis based on eye tracking and learner subjective perception. Computers in Human Behavior, 31,475-490. doi: 10.1016/j.chb.2013.04.022

Moreno, R. \& Durán, R. (2004). Do Multiple Representations Need Explanations? The Role of Verbal Guidance and Individual Differences in Multimedia Mathematics Learning. Journal of Educational Psychology, 96 (3), 492-503. doi: 10.1037/ 0022-0663.96.3.492

Moreno, R. \& Mayer, R. E. (1999). Cognitive principles of multimedia learning: The role of modality and contiguity. Journal of Educational Psychology, 91 (2), 358-368. http://dx.doi.org/10.1037/0022-0663.91.2.358

Nielsen, J. \& Pernice, K. (2010). Tecnicas de Eyetracking para usabilidad Web / Eyetracking Techniques for Web Usability. Anaya Multimedia-Anaya Interactiva.

Poole, A. \& Ball, L. J. (2006). Eye Tracking in Human-Computer Interaction and Usability Research: Current Status and Future Prospects. In Ghaoui, Claude (Ed.). Encyclopedia of Human Computer Interaction (pp. 211-219). Idea Group.

Pretorius, M. C., Calitz, A. P. \& Van, D. (2005). The added value of eye tracking in the usability evaluation of a network management tool. En Proceedings of the 2005 annual research conference of the South African institute of computer scientists and information technologists on IT research in developing countries (pp. 1-10). Pretoria: South African Institute for Computer Scientists and Information Technologists.

Slykhuis, D. A., Wiebe, E. N. \& Annetta, L. A. (2005). Eye-Tracking Students' Attention to PowerPoint Photographs in a Science Education Setting. Journal of Science Education and Technology, 14 (5-6), 509520. doi: 10.1007/s10956-005-0225-Z

Tobii (2014). [eye tracking]. [Consulta 28 de noviembre de 2014]. Recuperado de http:/ /www.tobii.com/.

Van, T. \& Scheiter, K. (2010). Eye tracking as a tool to study and enhance multimedia learning. Learning and Instruction, 20 (2), 95-99. doi:10.1016/j.learninstruc.2009.02.009

Walber, T., Neuhaus, C., Staab, S., Scherp, A. \& Jain, R. (2013). Creation of individual photo selections: read preferences from the users' eyes. In Proceedings of the 21st ACM international conference on Multimedia (pp. 629-632). doi: 10.1145/2502081.2502165

Ware, C. (2008). Visual thinking for design. Burlington, MA: Morgan Kaufmann.

Fecha de recepción: 25-06-2015 Fecha de evaluación: 06-09-2015

Fecha de aceptación: 07-10-2015 